\title{
Critical Susceptibility Exponent Measured from Fe/W(110) Bilayers
}

\author{
M.J. Dunlavy* and D. Venus \\ Dept. of Physics and Astronomy, McMaster University, \\ 1280 Main St. West, Hamilton Ontario, Canada
}

(Dated: November 1, 2018)

\begin{abstract}
The critical phase transition in ferromagnetic ultrathin $\mathrm{Fe} / \mathrm{W}(110)$ films has been studied using the magnetic ac susceptibility. A statistically objective, unconstrained fitting of the susceptibility is used to extract values for the critical exponent $\gamma$, the critical temperature $\mathrm{T}_{c}$, the critical amplitude $\chi_{o}$ and the range of temperature that exhibits power-law behaviour. A fitting algorithm was used to simultaneously minimize the statistical variance of a power law fit to individual experimental measurements of $\chi(\mathrm{T})$. This avoids systematic errors and generates objective fitting results. An ensemble of 25 measurements on many different films are analyzed. Those which permit an extended fitting range in reduced temperature lower than approximately $4.75 \times 10^{-3}$ give an average value $\gamma=1.76 \pm 0.01$. Bilayer films give a weighted average value of $\gamma=1.75 \pm 0.02$. These results are in agreement with the 2-dimensional Ising exponent $\gamma=\frac{7}{4}$. Measurements that do not exhibit powerlaw scaling as close to $\mathrm{T}_{c}$ (especially films of thickness $1.75 \mathrm{ML}$ ) show a value of $\gamma$ higher than the Ising value. Several possibilities are considered to account for this behaviour.
\end{abstract}

PACS numbers: 75.40.Cx, 77.80.Bh, 75.70.Ak

\section{INTRODUCTION}

Experiments that seek to measure critical phase transitions are very important to physics at a fundamental level. Careful experiments can be used to confirm or deny the theoretical models of universality and scaling. The true nature of the order parameter of a system, both in terms of dimensionality and degrees of freedom, is revealed at the transition and important physical insight is gained in the looking.

An ultrathin magnetic film closely approaches the physical realization of a truly two dimensional magnetic system, and offers a better system for studying critical phase transitions in two dimensions than more traditional layered bulk materials such as $\mathrm{Rb}_{2} \mathrm{CoF}_{4}{ }_{4}^{\frac{1}{\underline{ }}}$, where interlayer interactions will always be present, even if only to a small degree. Bander and Mills ${ }^{2}$ have shown that when ferromagnetic thin films have uniaxial anisotropy, the critical regime near the Curie temperature is described by the 2 dimensional Ising model. For this reason, a great number of measurements of the static critical exponents of ultrathin ferromagnetic films have been reported. Almost all of this experimental work concentrates on the critical exponent of the magnetization, $\beta 3$.4.5.6.7.8.9.10. To our knowledge, there are only a handful of reports in which the critical exponent of the magnetic susceptibility, $\gamma$, is investigated experimentally for an ultrathin magnetic film ${ }^{3.9 .11 .12 .13 .14}$. Unfortunately, almost all of these susceptibility studies have at least one of a number of deficiencies which call the results into question.

A common difficulty in the determination of critical exponents is the determination of $\mathrm{T}_{c}$. Small variations in the assumed value of $\mathrm{T}_{c}$ have a profound effect on the fitted value of the critical exponent, and introduce confidence limits that are usually much larger than those derived from a simple two parameter fit for the critical exponent and amplitude. The extreme sensitivity of the results to $T_{c}$ implies that the same data must be used to determine both $\mathrm{T}_{c}$ and the critical parameters. This is particularly true for metastable ultrathin films, since very small shifts in the critical temperature are often introduced by temperature cycling and annealing, or by residual vacuum contamination. A second difficulty is determining the temperature range where scaling is observed. Since real, finite systems do not show infinite divergences, the order parameter departs from power law behaviour close to $T_{c}$ because of finite size effects, dynamical effects (in ac measurements), a finite demagnetization factor, and so on. To fit the data properly, an objective, four parameter power-law fit of the data is required. In addition to $\mathrm{T}_{c}$ and $\gamma$, the fit should find values for the critical amplitude $\chi_{o}$ and the cutoff for power-law behaviour near $\mathrm{T}_{c}$. Finally, in order to demonstrate true systematic behaviour, it is clear that the analysis of many films and measurements is necessary.

It is perhaps surprising that after two decades of investigating the critical properties of ultrathin ferromagnetic films, that no published measurements of $\gamma$ meet

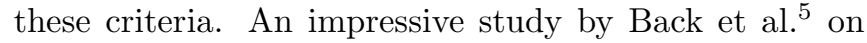
$\mathrm{Fe} / \mathrm{W}(110)$ ultrathin films determines $\beta$ and the exponent of the critical isotherm $\delta$ using the dc magnetization, and then derives $\gamma$ using the scaling relations between different exponents. The value of $\mathrm{T}_{c}$ is not fit, but rather taken to lie at the peak of the dc susceptibility for a particular experiment. The results agree with the predictions of the 2D Ising model. This represents a check of the internal consistency of the data and scaling relations, but is not an independent measurement of $\gamma$. Elmers et $\mathrm{al}^{\underline{3}}$ report dc susceptibility results for a series of submonolayer films of $\mathrm{Fe} / \mathrm{W}(110)$ and find $\gamma=$ $2.8 \pm 0.2$, significantly different than the $2 \mathrm{D}$ Ising value of $1.75^{15}$. It is not clear to what extent this finding is a result of using an incomplete film layer or if, as they suggest, the material is exhibiting the behaviour of an 
anisotropic Heisenberg system. Other studies report results only for a single measurement from a single film ${ }^{12}$. Still others use questionable criteria for determining $\mathrm{T}_{c}$, such as the disappearance of the imaginary component of the susceptibility in an ac measurement ${ }^{13}$, the peak of the real ac susceptibility ${ }^{14.16}$, or the presence of a "shoulder" above the peak of the susceptibility ${ }^{11}$ under special circumstances.

This paper presents the results of a collection of 25 measurements of the ac magnetic susceptibility of Fe films between 1.5 and 2.0ML grown epitaxially on $\mathrm{W}(110)$, and the values of $\gamma$ derived from them using an objective minimization of the statistical variance between the data and a power law fit using four parameters: $\mathrm{T}_{c}$, $\gamma$, the amplitude $\chi_{0}$, and the low reduced-temperature cutoff $\mathrm{t}_{x}$ for fitting. Error estimates on $\mathrm{T}_{c}$ and $\gamma$ are provided by $1 \sigma$ variations in the statistical $\chi^{2}$. The results fall into two distinct classes. Measurements exhibiting power law behaviour over a long range of reduced temperature extending down to a cutoff $t_{x}<4.75 \times 10^{-3}$ give an average critical exponent $\gamma=1.76 \pm 0.01$. Measurements which exhibit power law behaviour down to larger values of $\mathrm{t}_{x}$ show a systematic trend to higher values of $\gamma$ which depends rather linearly on $\ln \left(\mathrm{t}_{x}\right)$. The possibility that films which give a high value of $\gamma$ have a distribution of transition temperatures will be addressed to explain this unexpected result.

\section{THEORY}

According to scaling theory, the real component of the intrinsic magnetic susceptibility $\left(\chi_{i n t}=\partial M / \partial H\right)$ above the Curie temperature of a critical phase transition is described by the power-law equation:

$$
\chi_{\text {int }}^{\prime}(t)=\chi_{o} t^{-\gamma}
$$

where $\chi_{o}$ is the critical amplitude, $\gamma$ is the static critical exponent for the susceptibility of the order parameter and $t$ is the reduced temperature above $\mathrm{T}_{c}$, given as:

$$
t=\left(\frac{T-T_{c}}{T_{c}}\right) .
$$

For real measurements of the magnetic ac susceptibility, additional terms need to be added to account for both demagnetization and dynamical effects. The demagnetizing factor $\mathrm{N}$ is folded into the expression for the intrinsic susceptibility by augmenting the magnetic field by:

$$
H_{e f f}=H-N M
$$

where $H_{\text {eff }}$ is the effective field acting on the ferromagnet. This gives rise to an effective susceptibility of:

$$
\chi_{e f f}^{\prime}(T)=\frac{\chi_{i n t}^{\prime}(T)}{1+N \chi_{i n t}^{\prime}(T)} .
$$

It is easy to see that for a non-zero value of $N$, the susceptibility cannot diverge at $\mathrm{T}_{c} . N$ will "dampen" any experimental measurement of $\chi$ as long as the value of the product $N \chi_{i n t}$ is comparable to or greater than one.

To accurately describe results from ac susceptibility, it is necessary to add the effect of the relaxation time of the magnetization to the effective susceptibility. In the linear response approximation, for systems with an exponential relaxation time $(\tau)[\mathrm{M}(\mathcal{T}) \propto \exp (-\mathcal{T} / \tau)]$ where $\mathcal{T}$ is time under the influence of an externally applied sinusoidal field, the real dynamic susceptibility $\left(\chi^{\prime}\right)$ can be written as:

$$
\chi^{\prime}(T)=\frac{\chi_{\text {eff }}^{\prime}(T)}{1+(\omega \tau(T))^{2}}
$$

where $\omega$ is the driving frequency of the magnetic field 32 . This final form of the magnetic susceptibility limits the ability of experiments to probe critical behaviour very close to the transition. To observe any critical scaling in the experimental data, two requirements must be met: we must have $N$ sufficiently small and we must have $(\omega \tau)^{2}<<1.0$.

The extreme aspect ratio of ultrathin films leads to very small values of $N$. For systems that have their moments oriented in-plane, $N$ is proportional to first order to the thickness divided by the effective lateral dimension of the film 17 . For films that are one or two atomic layers thick and many thousands of lattice spacings wide, $N$ will be extremely small. This is another reason why ultrathin films are ideal for studies of critical phenomenon in two dimensions. Previous studies of the susceptibility on ultrathin films have attempted to estimate $N$ (and include the estimation in the power-law fits) by using the maximum value of the real susceptibility 13 . The argument proceeds by rearranging eq.(44) as follows:

$$
\frac{1}{\chi_{e f f}(T)}=\frac{1}{\chi_{\text {int }}(T)}+N
$$

This leads one to the conclusion that at $\mathrm{T}_{c}$, when $\chi_{\text {int }}$ is infinite, $N=1 / \chi_{\max }$. This simple treatment has several problems even for dc susceptibility measurements (where $\omega=0$ ) in that it ignores other effects (finite field, saturated correlation length, etc.) that will saturate the susceptibility and will give a value for $N$ that is artificially too high and is at best an upper limit $\frac{18}{}$. If this limit of $N$ was then used in the power-law analysis, the resulting quoted values for $\gamma$ should be called into question.

Dynamic effects are only significant near $\mathrm{T}_{c}$ where critical slowing down will lead to a large relaxation time for the equilibration of the order parameter near ${ }^{19}$. This can be less of a problem in dc measurements, but the increased signal-to-noise that is achieved in ac measurements make the effort to deal with the dynamics problem one worth undertaking. In fact, critical slowing effects should disappear once the temperature is increased more than a degree or two above $T_{c}$. Dynamic effects will change the temperature at which the susceptibility exhibits a maximum (depending on the measurement frequency used), making the evaluation of $\mathrm{T}_{c}$ by that method difficult if not impossible. 


\section{EXPERIMENT}

$\mathrm{Fe} / \mathrm{W}(110)$ ultrathin films with high quality epitaxial layers can be grown at least up to $2 \mathrm{ML}^{20}$. Previous studies of $\mathrm{Fe} / \mathrm{W}(110)$ have shown that the magnetic properties of the films depend sensitively on the film thickness 21 . There have been studies made on films less than $1.5 \mathrm{ML}$ that show interesting perpendicular magnetic behaviour due to the film structure which results from step-flow growth $^{22.23}$. For this study, we will concentrate on the thickness range from 1.5 to $2.0 \mathrm{ML}$ so as to ensure inplane magnetic behaviour.

The experiments were performed in an UHV environment with a base pressure of $1 \times 10^{-10}$ torr. The films were grown by molecular beam epitaxy (MBE) from a $99.995 \%$ pure iron wire. The substrate was a tungsten single crystal that had been cut and polished to expose the $[110]$ face. The cut is accurate to within $0.4^{\circ}$. The first layer was deposited at room temperature and then annealed for one minute to $500 \mathrm{~K}$. This slight annealing produces increased sharpness of the resulting pseudomorphic LEED pattern. Further depositions were performed at room temperature with no annealing. The film growth, thickness and quality were monitored by Auger electron spectroscopy (AES) and LEED.

The ac- $\chi_{m}$ measurements were made via the surface magneto-optic Kerr effect (SMOKE) using a focussed HeNe laser spot with a diameter of approximately $0.75 \mathrm{~mm}$. Small coils near the surface produced a sinusoidally oscillating magnetic field, $\mathrm{H}$, which influences the moments in the paramagnetic film above $\mathrm{T}_{c}$. The field was applied along the film's easy axis [1피. The surface magnetooptic Kerr effect produces a rotation of the polarization of the laser light reflected off of the magnetic surface. The signal manifests itself in changes in the light intensity at the photo-diode detector placed in front of the reflected laser beam. The $1 \mathrm{f}$ signal is read by a dualphase lock-in amplifier that can simultaneously record both the in-phase (or real) susceptibility $\left(\chi^{\prime}(\mathrm{T})\right)$ and the out-of-phase (or imaginary) susceptibility $\left(\chi^{\prime \prime}(\mathrm{T})\right)$. The raw signal is calibrated to SI units and the entire signal can be represented as

$$
\chi(T)=\chi^{\prime}(T)+i \chi^{\prime \prime}(T)
$$

Fig.(VI) shows a typical measurement of the complex susceptibility measured from a $1.8 \mathrm{ML}$ iron film. The measurement was made with an applied field amplitude of 0.7 Oersteds at a frequency of $400 \mathrm{~Hz}$.

It would be best to use an infinitesimally small field, but of course this is not possible experimentally. A study of magnetic susceptibility peak shape as a function of field was conducted to see what value of the field would give the best compromise between signal and finite field effects. It showed that the 'peak' shape of the susceptibility data measured in field amplitudes of 1.0 Oe and lower is not sensitive to the size of the field. Fig.(2) and (2b) show the maximum value and full-width, half- maximum (FWHM) for the susceptibility peaks respectively as a function of the amplitude of the applied field. The trend below 1.0 Oe in both graphs is stable, (except at extremely low fields where the signal itself disappears) but deviates for higher fields. Resulting measurements of a susceptibility peak measured in these small fields give a FWHM typically between two and three and a half degrees.In these measurements, smaller field amplitudes were accessible but this generally lead to a degradation of the signal-to-noise ratio.

Sample heating was accomplished by running ac current (no more than $1 \mathrm{~A}$ rms) through a small tungsten wire filament located behind the tungsten crystal. ACcurrent at $60 \mathrm{~Hz}$ was used to reduce the effects of stray offset fields at the surface. It had been found in the past that a dc-current introduced a 0.1 Oe offset field at the surface. The 0.10 field caused by the heating filament is much less than the applied field used in the measurement (typically 0.7Oe) and is much less than the field which increases the FWHM of the susceptibility peak (Fig[2). Any questions about the effect of the heating current were answered by comparing data taken while increasing and decreasing the film temperature respectively. The value of current used in the two methods differed by a factor of three, and there was absolutely no difference in the final data. The temperature of the film was measured using a W/WRh thermocouple embedded in the tungsten crystal and the rate of temperature increase/decrease was in most cases limited to $0.2 \mathrm{deg} / \mathrm{min}$. This low rate more than adequately compensates for thermal variations in the crystal and permits even heating of the film over the entire surface (app. $1 \mathrm{~cm}^{2}$ ).

\section{DATA ANALYSIS}

To fit the susceptibility data to eq.(1), an objective, many-parameter fit was used to determine the best values for the Curie temperature, $\mathrm{T}_{c}$, the critical exponent $\gamma$, the critical amplitude, $\chi_{o}$, and $t_{x}$ which is the smallest value of the reduced temperature to show power law scaling.

The fit is performed in double logarithm space $[\ln (\chi)$ vs $\ln (\mathrm{t})]$, the slope of which will correspond to the critical exponent. Taking the logarithm of the susceptibility necessitated the removal of data points where $\chi(\mathrm{T})$ goes to zero. Since these points are weighted the least in the fits [weighting in logarithmic space goes as $\left(1 / \chi_{d}^{2}\right)$ ], it is felt that this "weeding" out of points does not adversely affect the final fit. A small range of temperatures close to the peak was chosen for possible best values of $T_{c}$ used in the reduced temperature. For each considered value of $T_{c}$, a weighted least-squares fit was performed on the data in the new log-log data space from $\ln \left(t_{\max }\right)$ (which always corresponds to the data point measured at the highest temperature) to a cutoff value $\ln \left(t_{x}\right)$.

$t_{x}$ was itself varied over a range from just below $t_{\max }$ to a value of $t$ where the power-law scaling was obviously 
no longer valid. The variance of the fit was minimized for the best value of $T_{c}$ and the cut-off, $t_{x}$. The variance is the best test for a fit made in a many-parameter space 24 where the number of points does not remain constant. It is given by:

$$
s^{2}=\sum_{i_{t_{\max }}}^{i_{t_{x}}} \frac{\left(\ln \left(\chi_{i}\right)-F\left(t_{i}\right)\right)^{2}}{\sigma_{i}^{2}} / \sum_{i_{t_{\max }}^{i_{t_{x}}}} \frac{1}{\sigma_{i}^{2}}
$$

where $\chi_{i}$ is the $i^{t h}$ data point, $F\left(t_{i}\right)=\ln \left(\chi_{o}\right)+\gamma \ln (t)$ is the fitted function, and $\sigma_{i}$ is the error associated with the logarithm of each data point. The fitting algorithm generates a list of fitting results for all possible combinations of the inputs for the data in fig.(3). Fig.(4) shows a contour plot of $\mathrm{s}^{2}$ as a function of $\mathrm{T}_{c}$ and $\ln \left(\mathrm{t}_{x}\right)$. There is a global minimum at $\mathrm{T}_{c}=455.84 \mathrm{~K}$ and $\ln \left(t_{x}\right)=-5.355$ (corresponding to a temperature of $457.99 \mathrm{~K}$ ). There are local minima exhibited in the graphs that have higher values for $t_{x}$ than the global minimum. The fact that the global minimum fits the data closer to $\mathrm{T}_{c}$ increases its significance.

To get an error estimation on $\mathrm{T}_{c}$, the fits were recalculated while keeping the optimum value of $\ln \left(t_{x}\right)=-5.36$ to allow for a careful statistical $\chi^{2}$ analysis for a consistent number of data points. According to statistics for a multi-variable fit $^{24}$, the $65 \%$ confidence range for a fit value is judged by whatever range of the parameter corresponds to the limit where the value of the unreduced $\chi^{2}$ increases by one. Fig. (5) shows $\chi^{2}$ versus $\mathrm{T}_{c}$ for the data in fig.(3). Due to the good signal-to-noise of the data and the large number of points in the limited temperature range, the error for $\mathrm{T}_{c}$ is very small. The number of points in the fit used for Fig 5 is 1905 , which will give a reduced $\chi^{2}$ for the fit of 1.8 , signifying a very good fit to the data. The $\mathrm{T}_{c}$ value from this analysis is $455.84 \pm 0.03$. While this range of $\mathrm{T}_{c}$ creates an uncertainty in $\gamma$ on the order of the error from the least-square analysis, the two effects should compound to increase the confidence limit on $\gamma$ slightly. The value for the critical exponent from the particular data set in fig. (3. 1 ) is $\gamma=1.75 \pm 0.02$. The fitted critical amplitude $\chi_{o}$ is $7.3 \pm 0.3 \times 10^{-3}$.

It now becomes necessary to check for both dynamic and demagnetization effects in the data. It has already been remarked that a demagnetization factor equal to $1 / \chi_{\max }$ provides an upper limit on the value of $N$. This assumption would lead to a value of $N$ for the data in fig. (3) to be about $1 / 150$ or $6.67 \times 10^{-3}$. According to eq.(4), once the value of the product $N \chi$ approaches 0.05 , the observed power law behaviour of the intrinsic susceptibility is lost. For this data set, this would occur at a temperature of $464.2 \mathrm{~K}$. This would give a value of $\ln \left(\mathrm{t}_{x}\right)$ approximately equal to -4.0. In other words, if we believe the above estimate for $N$, then no linear segment in double-log space would extend closer to $\mathrm{T}_{c}$ than this. The results in fig.(3) clearly show the linear segment extending much lower than -4.0. The value of $N$ must therefore be much smaller. The power-law behaviour in fact deviates at a temperature of approximately $458.0 \mathrm{~K}$. If we take the ' $5 \%$ rule' a step further, the maximum value of $N$ then becomes approximately $1 / 1632$ or $6.1 \times 10^{-4}$, a full order of magnitude lower than the previous estimate. This lower value is more in keeping with the value of $N$ expected from geometric arguments and provides a new upper limit on $N$.

Checking the saturation from dynamic effects requires a more definite knowledge of the time response of the moments as a function of temperature than these measurements currently allow. However, a simple calculation can be made on a theoretical basis. Near $\mathrm{T}_{c}$, the relaxation time of the magnetization will undergo critical slowing down which, by theory, follows the formula:

$$
\tau(T)=\tau_{o} t^{-z \nu}
$$

where $\nu$ is the critical exponent associated with the correlation length and $z$ is the critical slowing down exponent. While there are very few experiments that measure the critical slowing down of the relaxation time, $\tau$, on ferromagnetic systems, theoretical simulations ${ }^{25.26}$ suggests that the value of $z$ should be approximately 2.2 for the $2 \mathrm{D}$ Ising system. The value for $\tau_{o}$ should be very small, on the order of inverse- $\mathrm{GHz}$ to agree with FMR resonance frequencies.

To see no dynamic effect interference in $\chi_{i n t}$ as per eqn.(5), you need $\omega \tau$ less than 1.0. If we apply the ' $5 \%$ ' rule again and use $z \nu=2.2, \tau_{o}=1 \times 10^{-9} \mathrm{~s}$ and $\omega=(2 \pi) 150.0 \mathrm{~Hz}$, we find that $\ln \left(t_{x}\right)$ will be -5.6 . This is close to the fit value for $\ln \left(t_{x}\right)$ and may be the reason for the saturation of the susceptibility. Better estimates of $\tau_{o}$ and $z \nu$ are required to pursue this question further.

\section{RESULTS FROM MANY FILMS}

Critical power-law fitting was performed on a sample of 25 different measurements from many films grown between 1.5 and 2.0 ML. The results show that the value of $\gamma$ that comes from the fit is dependent on the thickness of the film. Fig.(6) shows a plot of $\gamma$ as a function of $\ln \left(t_{x}\right)$ for all 25 measurements. It is apparent from the plot that $\gamma$ is not only thickness dependent but that there is also the presence of an extra effect which causes films with a higher value of $\gamma$ to fit $t_{x}$ further from $\mathrm{T}_{c}$.

The following weighted and unweighted average results for $\gamma$ can be given: (1) For bilayer films, the weighted average value of $\gamma$ is $1.75 \pm 0.015$ with an unweighted average of $1.74 \pm 0.023$ (2) For sesqilayer films, the weighted average is $1.63 \pm 0.01$. (This weighted average is suspect as there are only 3 data points with small individual error which do not overlap). The unweighted average is $1.68 \pm 0.13$. (3) The weighted average value of gamma for films with $\ln \left(\mathrm{t}_{x}\right)$ less than -5.35 is $1.76 \pm .01$. The unweighted average is $1.76 \pm 0.04$. Most of the films with values of $\mathrm{t}_{x}$ in this last range are either 2.0 or $1.5 \mathrm{ML}$, but it should be stated that there is also one measurements at $1.75 \mathrm{ML}$ and one other just below $1.5 \mathrm{ML}$. 
Films with a thickness of 2.0ML and 1.5ML consistently have the lowest values of $\ln \left(\mathrm{t}_{x}\right)$ and these are the films that give (on average) the $2 \mathrm{D}$ Ising result. There is no supporting evidence to the idea that all of these measurements would show Ising behaviour if the linear fit was performed on a range of data closer to $\mathrm{T}_{c}$. An examination of the fitted value of $\gamma$ as a function of $t_{x}$ for individual measurements show that there is absolutely no increase in $\gamma$ with increasing $t_{x}$. It is thought that the higher values of $t_{x}$ are an indicator of another as-yet not understood process that affects the power law scaling when the film thickness is just below or equal to 1.5 and $2.0 \mathrm{ML}$. It is possible that this process is also responsible for the high value of $\gamma$ reported for films of $0.8 \mathrm{ML}$ thickness ${ }^{3}$.

We have examined several possible explanations for this behaviour. The first involved using corrections to scaling arguments 27.28 that should be taken into account for fitting data far away from $\mathrm{T}_{c}$. If this is the case, then the effective value of the exponent, $\gamma_{e f f}$ is approximated by

$$
\gamma_{e f f}=\gamma-a \Delta|\bar{t}|^{\Delta},
$$

where $a$ is a constant, $\bar{t}$ is some "average temperature" representing the fitting range, and the exponent $\Delta$ is close to 0.5 for Ising systems ${ }^{27}$ regardless of the dimensionality of the system. If $t_{x}$ is chosen as $\bar{t}$, the data in fig.(6) can be reasonably described by eq.(10) with $\Delta \approx 3$. The large discrepancy between the fitted and theoretical value of $\Delta$ suggests that corrections to scaling are not the important factor here.

Another possibility for the rising value of $\gamma$ is the idea of dimensional crossover from an Ising system to an anisotropic Heisenberg system as a function of temperature ${ }^{29}$. It is well known that true twodimensional Heisenberg systems cannot support long range magnetic order above $\mathrm{T}=0$ 年, but only a small amount of anisotropy is required to lift this restriction 2 . It is possible that the films that exhibit high values of $\ln \left(t_{x}\right)$ are showing higher values of $\gamma$ due to a change in the anisotropy. This explanation was also offered by Elmers et.al $\stackrel{3}{\underline{3}}$ for their $0.8 \mathrm{ML}$ results, and it is interesting to note that the value of $\ln \left(\mathrm{t}_{x}\right)$ in their result would be -5.3 , which is consistent with the onset of high $\gamma$ values in this study. However, there are several reasons against this idea. First, none of the data with an Ising exponent show a crossover to larger $\gamma$ when $t_{x}$ is artificially increased. Second, none of the data used in this work, including those data sets that fit with a $\ln \left(\mathrm{t}_{x}\right)$ value less than -6 , shows anything resembling a 'break point' in the double-log slope indicating different critical power law over different temperature ranges, which would provide a clear indication that dimensional crossover is occurring. Finally, a reduced anisotropy should result in a change in the trend of the transition temperature as a function of thickness 31 , an effect which we do not observe.

The third possibility is that the films in the sensitive thickness range have a wide distribution of transition temperatures. This effect on the fitted slope is easy to understand. If some fractional area of the film undergoes a phase transition at a temperature slightly above the average "mean" value of $\mathrm{T}_{c}$ used to reduce the temperature for the logarithmic plot, then those areas will register as an artificially high slope in the fit. While the exact nature of the distribution is unknown, it is certain that any distribution with values above the $\mathrm{T}_{c}$ used in the fitting routine will increase the fitted exponent. To gauge the effect quantitatively, a series of data sets were modeled using a normalized Gaussian distribution of $\mathrm{T}_{c}$ and an intrinsic value of $\gamma$ of 1.75 . It was found that it takes a half-width of just over $0.5 \mathrm{~K}$ to cause a $1 \%$ increase in $\gamma$. No significant increase is found to occur as long as the half-width is less than $0.25 \mathrm{~K}$. A half-width of $1 \mathrm{~K}$ gives a fit exponent of 1.81 , a $3.5 \%$ increase. To achieve a fit value for $\gamma$ of 3 (near the maximum fit value in the 25 measurements we fit), requires a half-width of $2.5 \mathrm{~K}$. It may be that the films less than $1.5 \mathrm{ML}$ and between 1.5 and 2.0ML are more sensitive to small structural inhomogeneities that give rise to a wider distribution of $\mathrm{T}_{c}$. Films with a complete second monolayer will be more homogeneous than films that are slightly thinner. We speculate that the distribution of transition temperatures may be related to the distribution of atoms that are located at step edges between the first and the incomplete second monolayer. For the complete $2 \mathrm{ML}$, the films should be very homogeneous and a narrow distribution may be expected. The 1.5ML films have equal areas that are $1 \mathrm{ML}$ and $2 \mathrm{ML}$ thick respectively and as such present a uniform configuration of steps which have been shown $^{23}$ to give a correlated magnetic state. The 1.75 films are on the threshold of the percolation limit of the second monolayer and it is possible that slight structural deviations are more likely to cause a wider distribution of transition temperatures. This suggestion may also go towards explaining the $0.8 \mathrm{ML}$ results quoted above ${ }^{3}$.

\section{CONCLUSION}

We report the results of fitting measurements of the magnetic ac-susceptibility for critical power law exponents. We find that the critical exponent for bilayer $\mathrm{Fe} / \mathrm{W}(110)$ films to b e $1.75 \pm 0.02$ and for films in general with a value of $t_{x}$ below $4.75 \times 10^{-3}, \gamma=1.76 \pm 0.01$. This result confidently place this system in the 2D Ising universality class. The fitting routine allows the simultaneous extraction of the critical exponent and the critical temperature from a single measurement of the susceptibility. There is evidence of another process which affects fitting of the susceptibility for certain thicknesses. This may be due to these films having a larger distribution of critical temperatures. 


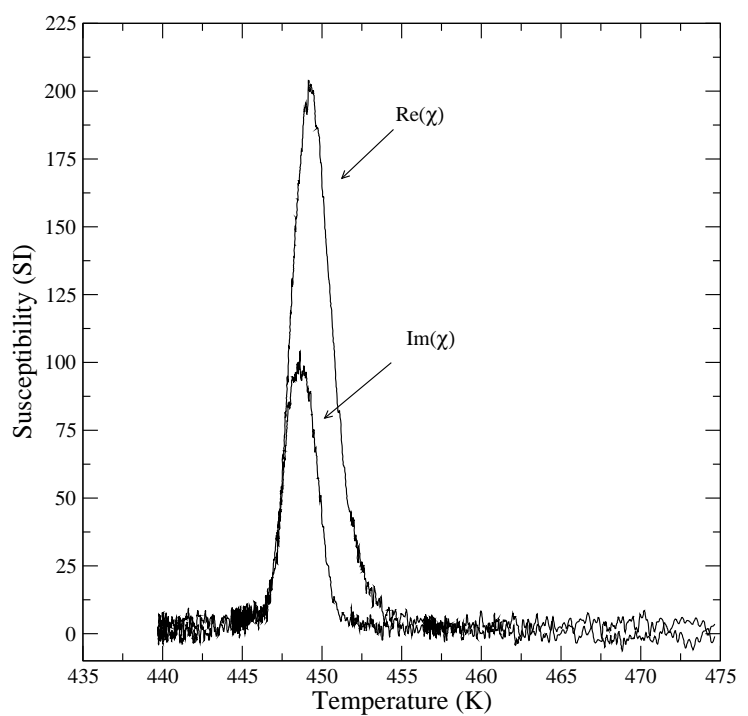

FIG. 1: Magnetic ac-susceptibility measured from a $1.8 \mathrm{ML}$ film of iron grown upon W(110). The real and imaginary components of the susceptibility were measured simultaneously.

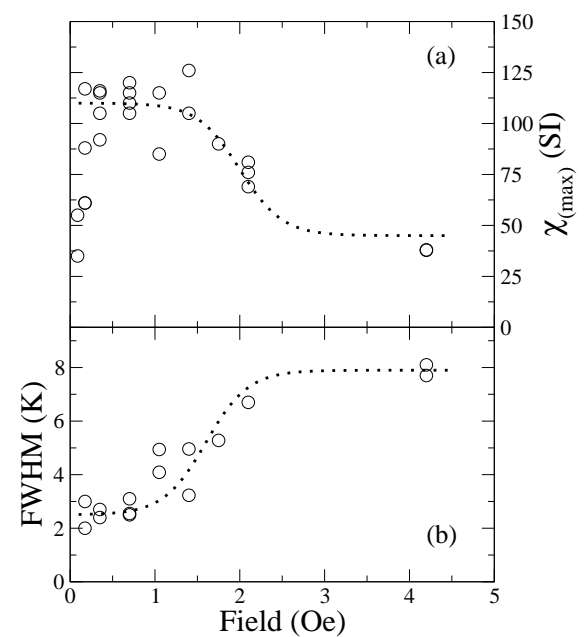

FIG. 2: (a) The maximum value of the magnetic susceptibility as a function of applied magnetic field. (b) FWHM of the real susceptibility peak plotted as a function of applied field amplitude. The minimum half-width is achieved for fields less than 1 Oersted.

\section{Acknowledgments}

The authors wish to acknowledge the many technical contributions made by Marek Kiela. This work was supported by the National Science and Engineering Research Council of Canada.
* Electronic address: dunlavmj@mcmaster.ca

1 E. J. Samuelson, Phys. Rev. Lett. 31, 936 (1973).

2 M. Bander and D. L. Mills, Phys. Rev. B 38, 12015 (1988).

3 H. J. Elmers, J. Hauschild, and U. Gradmann, Phys. Rev. B 54, 15224 (1996).
${ }^{4}$ Y. Li and K. Baberschke, Phys. Rev. Lett 68, 1208 (1992).

${ }^{5}$ C. H. Back, C. Würsch, A. Vaterlaus, U. Ramsperger, U. Maler, and D. Pescia, Nature 378, 597 (1995).

6 C. A. Ballentine, R. L. Fink, J. Araya-Pochet, and J. L. Erskine, Applied Phys. A 49, 459 (1989). 

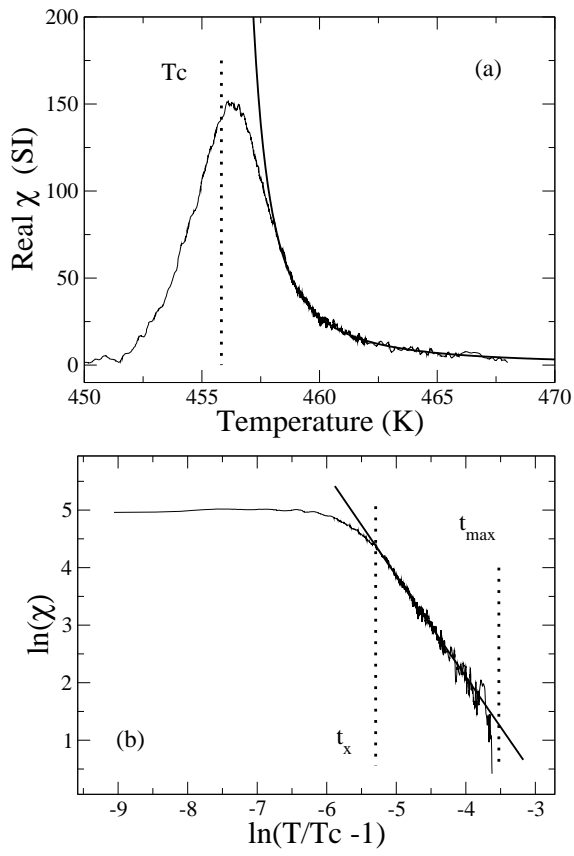

FIG. 3: Power law fit for a typical susceptibility measurement. (a) $\chi$ versus temperature. Solid line shows the fit and dotted line shows position of $\mathrm{T}_{c}$. (b) Fit in log-log space, with dotted lines showing position of $t_{\max }$ and $\mathrm{t}_{x} \cdot \mathrm{t}_{\max }$ always corresponds to the maximum temperature which was measured.

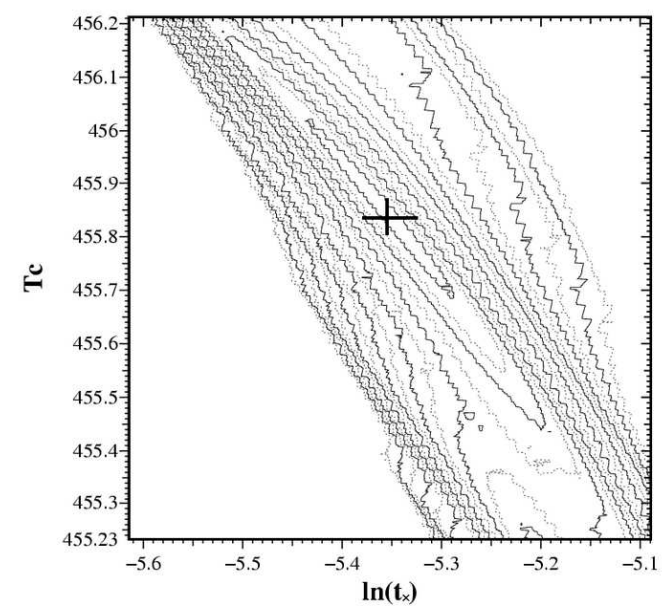

FIG. 4: Contour plot of $\mathrm{s}^{2}$ as a function of $\mathrm{T}_{c}$ and $\ln \left(t_{x}\right)$. The global minimum (indicated by the cross) shows the values of $\mathrm{T}_{c}=455.84$ and $\ln \left(t_{x}\right)=-5.355$ corresponding to the best fit.

7 F. Huang, M. T. Kief, G. J. Mankey, and R. F. Willis, Phys. Rev. B 49, 3962 (1994).

8 Y. Li, M. Farle, and K. Baberschke, Phys. Rev. B 41, 9596 (1990).

9 H. J. Elmers, J. Hauschild, G. H. Liu, and U. Gradmann, J. Appl. Phys. 79, 4984 (1996).

10 J. Kohlhepp, H. J. Elmers, S. Cordes, and U. Gradmann,

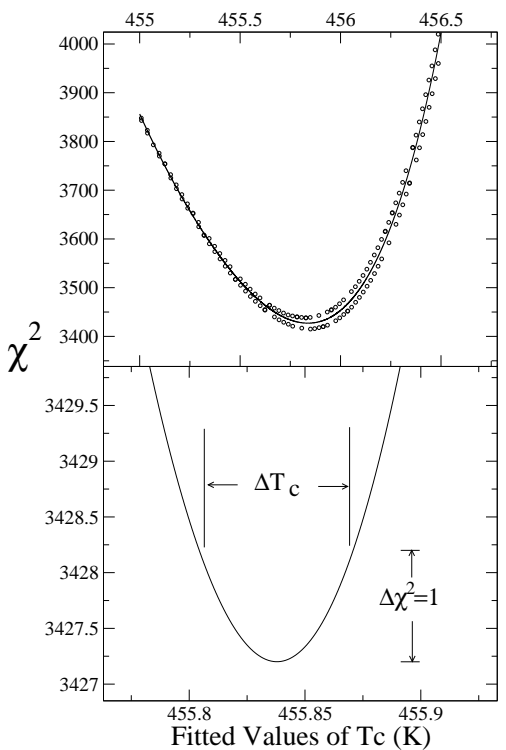

FIG. 5: Graphs of $\chi^{2}$ versus value of $\mathrm{T}_{c}$ used in fit. Fig.(a) shows the minimum in $\chi^{2}$ with a smooth function fit to the points. Fig.(b) shows the fitted curve with an indicated range corresponding to a change in $\chi^{2}$ of 1.0 . The value of $\mathrm{T}_{c}$ with error for this data set is $\mathrm{T}_{c}=455.84 \pm .03 \mathrm{~K}$

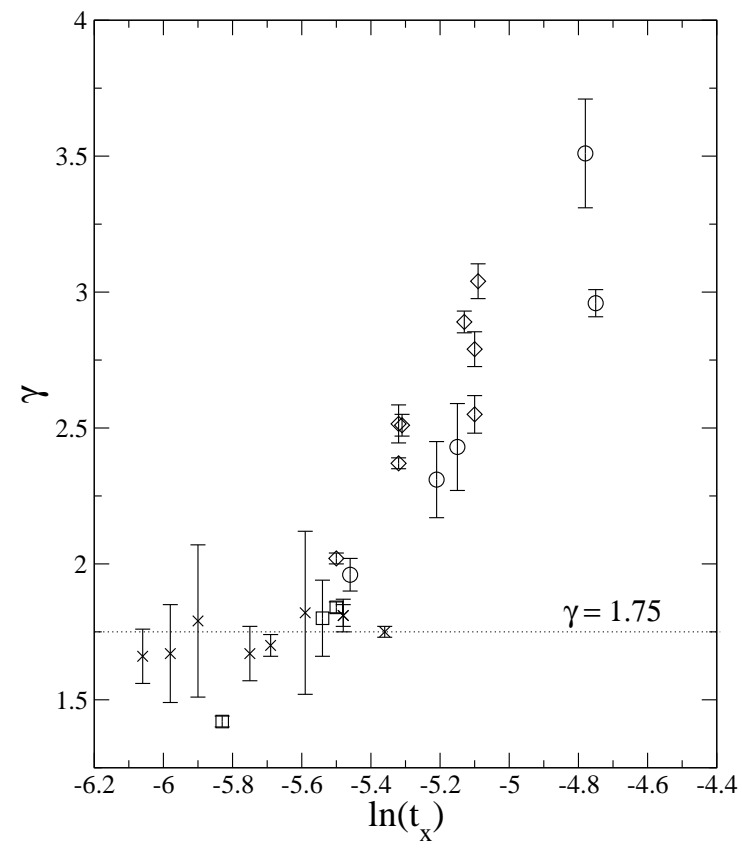

FIG. 6: Best fit values of $\gamma$ plotted as a function of reduced temperature cutoff, $\ln \left(\mathrm{t}_{x}\right)$. Open circles represent films that are slightly less than $1.5 \mathrm{ML}$, squares are $1.5 \mathrm{ML}$ films, diamonds are $1.75 \mathrm{ML}$, and X's are $2.0 \mathrm{ML}$. 
Phys. Rev. B 45, 12287 (1992).

11 U. Bovensiepen, C. Rudt, P. Poulopoulos, and K. Baberschke, JMMM 231, 65 (2001).

12 C. S. Arnold, M. J. Dunlavy, and D. Venus, Rev. Sci. Inst. 68, 4212 (1997).

13 C. Rudt, P. Poulopoulos, J. Lindner, A. Scherz, H. Wende, K. Baberschke, P. Blomquist, and R. Wappling, Phys. Rev. B. 65, 220404(R) (2002).

14 U. Stetter, M. Farle, K. Baberschke, and W. G. Clark, Phys. Rev. B 45, 503 (1992).

15 L. Onsager, Phys. Rev. 65, 117 (1944).

16 G. Garreau, M. Farle, E. Beaurepaire, and K. Baberschke, Phys. Rev. B 55, 330 (1997).

17 S. Chikazumi, Physics of Magnetism (John Wiley \& Sons, 1964).

18 A. Aspelmeier, M. Tischer, M. Farle, M. Russo, K. Baberschke, and D. Arvanitis, JMMM 146, 256 (1995).

19 P. C. Hohenberg and B. I. Halperin, Rev. Mod. Phys. 49, 435 (1977).

${ }^{20}$ U. Gradmann and G. Waller, Surf. Sci. 116, 539 (1982).

${ }^{21}$ H. J. Elmers, J. Hauschild, H. Fritzsche, G. Liu, U. Gradmann, and U. Kohler, Phys. Rev. Lett. 75, 2031 (1995).

22 J. Hauschild, U. Gradmann, and H. J. Elmers, Applied Phys. Lett. 72, 3211 (1998).
23 H. J. Elmers, J. Hauschild, and U. Gradmann, Phys. Rev. B 59, 3688 (1999).

24 P. Bevington and D. Robinson, Data Reduction and Error Analysis for the Physical Sciences (McGraw-Hill Inc., 1992).

25 M.-D. Lacasse, J. Vinals, and M. Grant, Phys. Rev. B 47, 5646 (1993).

26 J. Wang, Phys. Rev. B 47, 869 (1993).

27 N. Goldenfeld, Lectures on Phase Transitions and the Renormalization Group (Addison-Wesley Publishing Co., 1992).

28 M. D. Lumsden, B. D. Gaulin, and H. Dabkowska, Phys. Rev. B 57, 14097 (1998).

29 K. Binder and D. P. Landau, Phys. Rev. B 13, 1140 (1976).

30 N. D. Mermin and H. Wagner, Phys. Rev. Lett. 17, 1133 (1966).

31 R. P. Erickson and D. L. Mills, Phys. Rev. B 43, 11527 (1991).

32 In these equations, the relaxation time of the magnetization is used, where $\frac{d M}{d t}=-\frac{1}{\tau}\left(M-M_{\infty}\right)$. Dissipation can also be expressed in terms of the relaxation of the effective field using the Landau-Liftshitz equation and the damping parameter. The two terms are related by $\tau=\frac{\chi_{e f f}}{\lambda}$. 